\title{
Bacterial Agents Causing Food Poisoning among Patients Attending Thika Level 5 Hospital, Kiambu County, Kenya
}

\author{
Karanja N. Esther ${ }^{1}$, Menza C. Nelson'2, Kabiru W. Ephantus ${ }^{1}$ \\ ${ }^{1}$ Department of Community Health and Epidemiology, Kenyatta University, Nairobi, Kenya \\ ${ }^{2}$ Department of Medical Laboratory Sciences, Kenyatta University, Nairobi, Kenya \\ Email: evasalehina@gmail.com,menza.nelson@ku.ac.ke, kabiru.ephantus@ku.ac.ke
}

How to cite this paper: Esther, K.N., Nelson, M.C. and Ephantus, K.W. (2019) Bacterial Agents Causing Food Poisoning among Patients Attending Thika Level 5 Hospital, Kiambu County, Kenya. Open Journal of Medical Microbiology, 9, 168-177.

https://doi.org/10.4236/ojmm.2019.94016

Received: September 7, 2019

Accepted: November 30, 2019

Published: December 3, 2019

Copyright $\odot 2019$ by author(s) and Scientific Research Publishing Inc. This work is licensed under the Creative Commons Attribution International License (CC BY 4.0).

http://creativecommons.org/licenses/by/4.0/

\begin{abstract}
Food poisoning is caused by the ingestion of contaminated food or water that contains microbial agents that cause illness or release their toxins onto the food. Examples of these microbial agents are Staphylococcus aureus, Listeria monocytogens, Clostridium botulinum, Salmonella typhiridium, and Salmonella enteritidis. Epidemiological monitoring of food poisoning is highly desirable for continuous determination of the existing causative species and disease trends. The study was conducted at Thika level 5 hospital. The objective of the study was to establish the prevalence of food poisoning at Thika level 5 hospital. A cross-sectional study design and purposive sampling technique was adopted in this study. Interview scheduled questionnaires and collection of stool samples from in-patients presenting with suspected food poisoning at Thika level 5 , hospital was used for data collection. Stool culture tests were used to identify the bacterial causative agents of food poisoning. Statistical package for social sciences (SPSS) was used in the analysis of the data. Ethical approval was sought from Kenyatta University Ethical Review Committee, the Ministry of Education Science and Technology and Kiambu County Government. Informed consent was sought from the respondents after they had been informed about the study. The study findings showed that of the sample taken 28 (26.4\%) were positive of Cholera while 78 (73.6\%) were positive of Salmonella. The study recommends early set-up of emergency wards to contain outbreaks of infectious diseases together with well-equipped diagnostic labs for prompt response.
\end{abstract}

\section{Keywords}

Bacteria, Food Poisoning, Salmonella 


\section{Introduction}

Food poisoning is a significant public health problem as well as endemic worldwide and is one of the most common reasons for visiting accident and emergency department (EDs), [1] Millennium development goal number one which is to eradicate extreme poverty and hunger [2] is being directly compromised due to food borne illnesses [3]. This is because money is spent on medical costs instead of development and days are lost due to illness and disability. Food borne diseases (FBD) are defined by the world's health organization as diseases of infectious or toxic nature caused by the consumption of food or water [4]. The symptoms vary widely depending on the etiological agents. Common symptoms usually include abdominal pains, diarrhea, vomiting, mild fever, headache and vomiting [4]. In 2010 for global estimates, there were 600 (95\% uncertainty interval [UI] (0.5 confidence interval) 410 - 950) million food borne illnesses and 420,000 (95\% [UI] uncertainty interval (0.5 confidence level) 310,000 - 600,000) deaths [5]. Mortality in Africa is estimated to be at around 750,000 persons per year in all ages. Disability adjusted life years lost to food borne and waterborne disease is estimated to be $4.1 \%$ globally compared to $5.1 \%-7.1 \%$ in the African region [5]. Acute food borne intoxications and diseases are of more concern to governments and the food industry today because of the increasing number of large outbreaks being reported, the aging population are immune-compromised and re-emerging resistant bacterial strains [6]. Food poisoning mostly affects people with poor hygiene practices. The reason for this study was to establish the prevalence of microbial food poisoning in Thika level 5 hospital because a lot of studies concerning microbial contamination have been done but the etiology agents are unknown. Food borne illnesses are concerned with some strains of resistant bacteria that are re-emerging worldwide such as Methicillin resistant Staphylococcus aureus (MRSA) that places a huge added cost to healthcare together with an increasingly vulnerable population leading to more frequent medical and surgical complications and greater mortality like colectomy. This then makes it important to monitor incidence, enhance surveillance method, identify the most vulnerable age group at risk, and characterize the molecular epidemiology of the strain causing food borne illness [7]. The study was conducted in Thika level 5 hospital because of inadequate investigation where it is not routine to isolate the causative organism. The results will be used by policy makers to improve the diagnosis of food poisoning [8]. The study set out to determine the prevalence of food poisoning at Thika level 5 hospital in Kiambu county as well as the bacterial causative agents associated with food poisoning at Thika level 5 hospital.

\section{Material and Methods}

\subsection{Study Area}

The study was conducted at Thika level 5 in Kiambu County, Kenya. This study was conducted between December 2017 and December 2018. The hospital is lo- 
cated in Thika town in Kiambu County of Kenya and it receives many referral patients from the neighboring rural areas in Kiambu County.

\subsection{Study Design}

A cross sectional study design was adopted among in-patients presenting with suspected food poisoning in Thika level five hospitals.

\subsection{Study Population}

Patients admitted in Thika level 5 hospitals in Kiambu County Kenya presenting with suspected food poisoning were enrolled in the study. The age criteria were a factor which was put into consideration because of informed consent.

\subsection{Sampling Technique}

Purposive sampling technique was used in enrollment of participants to be included in the study. Consecutive patients were recruited until the sample size was achieved.

The sample size was determined by the formula of (Rosner, 2010)

$$
N=\frac{\varrho^{2}\left(Z_{1-\beta}+Z_{1-\alpha}\right)}{\mu_{0}-\mu_{1}}
$$

where $\varrho$ represents standard deviation of common medium of culture and that of differential media culture.

Mean of the sample is represented by $\mu_{0}$.

Mean of the population is represented by $\mu_{1}$.

$$
\begin{gathered}
\varrho^{2}=1.71^{2}, Z_{1-\beta}=1.645, Z_{1-\alpha}=1.96 \\
\mu_{0}=27.1, \mu_{1}=26.5
\end{gathered}
$$

$(1-\beta)-\beta$-represents power at 95\% (as 90\% is suitable for field work).

$(1-\alpha)-\alpha$-represents confidence at $95 \%$ (using two tailed distribution table).

A minimum number of 106 patients will be required.

\subsection{Study Approval, Ethical Consideration and Informed Consent}

Approval was sought from Kenyatta university ethical review committee (KUERC). Authority was sought from NACOSTI. Clearance and permission was sought from Thika level 5 hospital. Confidentiality, anonymity and informed consent were observed.

\subsection{Laboratory Analysis}

\subsubsection{Sample Collection}

Stool was collected in polypot containers through the defecation process. In case a patient was not able to produce stool swabbing was done. The specimen was taken to the laboratory for analysis.

\subsubsection{Vibrio Cholera \& Salmonella Identification}

The $S$. typhi antigen rapid test cassette was used for the rapid detection of $S$. 
typhi antigens in human feces [9]. The vibrio cholera O1/O139 antigen combo rapid test was used for the qualitative, presumptive detection of vibrio cholera $\mathrm{O} 1$ and or O139 in human fecal specimens [10].

MacConkeyagar is a selective and differential culture medium that was used to selectively isolate Gram-negative and enteric bacilli and differentiate them based on lactose fermentation. TCBS agar is highly selective for the isolation of $V$. cholerae. TCBS-Thiosulphate-citrate-bile-salts agar medium was used for detection of $V$, cholerae antigens in human feaces.

\subsection{Data Analysis}

Shapiro-Wilks statistical tests analysis was run on characteristics of populations. A further Kruskall Wallis $\mathrm{H}$ test was used to compare the differences in the median of the two groups. Chi square was further employed to check for the relationship between socio-demographic and socioeconomic characteristics of the patients and diarrhea as a sub-set of food poisoning.

\section{Results}

\subsection{Socio-Demographic, Socio-Economic Characteristics of Patients in Kiambu County}

In this study, 107 participants were recruited. Out of 107 participants, males were $65(60.7 \%)$ while $42(39.3 \%)$ were females. The number of male participants were significantly higher compared to females and were associated with diarrhea $\left(\chi^{2}=1.562, \mathrm{p}=0.393, \mathrm{df}=1\right) . \mathrm{P}=0.393$ was higher than $\mathrm{p}=0.05$, chi-square statistics indicates there was no significant association between sex and diarrhea symptoms (Table 1).

Most of the respondents in this study were single 61 (57.0\%) while 46 (43.0\%) were married $\left(\chi^{2}=0.761, p=0.383, d f=1\right) \cdot p=0.383$ was higher than $p=0.05$, chi-square statistics indicates there was no significant relationship between level of education and diarrhea symptoms (Table 1).

Most of the patients who had diarrhea were in the age group 21 - 40 years with $56(52.8 \%)\left(\chi^{2}=9.792, \mathrm{p}=0.280, \mathrm{df}=3\right)$. The second age group was $\leq 20$ years with $21(19.8 \%)$ and it was followed by age group $61-80$ years at 20 $(18.6 \%)$ and finally age group $41-60$ at $9(8.5 \%) . \mathrm{P}=0.280$ was higher than $\mathrm{p}=$ 0.05 , chi-square statistics indicates there was no significant association between age and diarrhea symptoms (Table 1 ).

Most of the respondents that were affected were from the slums 46 (43.0\%) and were therefore statistically significant, followed by those from Peri-urban 36 (33.5\%). From the urban we had $21(19.5 \%)$ and lastly the rural $4(3.7 \%) .\left(\chi^{2}=\right.$ 25.993; $\mathrm{df}=3 ; \mathrm{p}=0.049) . \mathrm{P}=0.049$ was less than $\mathrm{p}=0.05$, chi-square statistics indicates there was an association between residence and diarrhea symptoms (Table 1).

Most of the respondents in this study had attained tertiary level of education $54(50.5 \%)$. It was followed by secondary level of education with 37 (34.5\%). 
Table 1. (a) Socio-demographic characteristics of respondents with diarrhea; (b) Socio-economic characteristics of respondents with diarrhea.

(a)

\begin{tabular}{ccccc}
\hline Category & Frequency & Percentage & P-value & Significance \\
\hline Sex & 65 & 60.7 & $\mathrm{P}=0.393$ & $\chi^{2}=25.1 .563$ \\
Male & 42 & 39.3 & & $\mathrm{df}=1$ \\
Female & & & & \\
Marital status & 61 & 57.0 & $\mathrm{P}=0.383$ & $\chi^{2}=0.761$ \\
Single & 46 & 43.0 & & $\mathrm{df}=1$ \\
Married & & & & \\
Age & 21 & 19.8 & $\mathrm{P}=0.280$ & $\chi^{2}=9.792$ \\
$\leq 20$ & 56 & 52.8 & & $\mathrm{df}=3$ \\
$21-40$ & 09 & 8.5 & & \\
$41-60$ & 20 & 18.6 & & \\
$61-80$ & & & & \\
Residence & 21 & 19.5 & & \\
urban & 36 & 33.5 & $\mathrm{P}=0.049$ & $\chi^{2}=25.993$ \\
Peri-urban & 4 & 3.7 & & $\mathrm{df}=3$ \\
rural & 46 & 43.0 & & \\
slum & & & & \\
\hline
\end{tabular}

Significant at $5 \% .{ }^{*}$ two-tailed distribution.

(b)

\begin{tabular}{ccccc}
\hline Category & Frequency & Percentage & P-value & Significance \\
\hline Education level & & & & \\
Primary & 16 & 15.0 & & \\
Secondary & 37 & 34.5 & $\mathrm{P}=0.609$ & $\chi^{2}=0.991$ \\
Tertiary & 54 & 50.5 & & $\mathrm{df}=2$ \\
Occupation & & & & \\
Business owner & 21 & 19.8 & & \\
Casual laborer & 9 & 8.5 & $\mathrm{P}=0.0009$ & $\chi^{2}=16.992$ \\
Petty trader & 6 & 5.6 & & \\
Retired & 9 & 8.5 & & $\mathrm{df}=5$ \\
Salaried & 29 & 27.3 & & \\
Unemployed & 33 & 30.1 & & \\
Economic status & & & & \\
$<2000 \mathrm{k}$ & 3 & 2.8 & & \\
$2000 \mathrm{k}-10,000 \mathrm{k}$ & 15 & 14.0 & $\mathrm{P}=0.0812$ & \\
$10,000 \mathrm{k}-30,000 \mathrm{k}$ & 33 & 30.8 & & \\
$30,000 \mathrm{k}-60,000 \mathrm{k}$ & 31 & 29.0 & & \\
$60,000 \mathrm{k}-150,000 \mathrm{k}$ & 21 & 19.6 & & \\
$150,000 \mathrm{k}-200,000 \mathrm{k}$ & 4 & 3.7 & & \\
\hline
\end{tabular}

Significant at $5 \% .{ }^{*}$ two-tailed distribution. 
Lastly those with primary level of education were $16(15 \%) \cdot\left(\chi^{2}=0.991, \mathrm{p}=\right.$ $0.609, \mathrm{df}=2) . \mathrm{p}=0.609$ was higher than $\mathrm{p}=0.05$ chi-square statistics indicates there was no significant association between level of education and diarrhea symptoms (Table 1).

Most of the respondents were unemployed 33 (30.1\%) and were statistically significant followed by salaried at $29(27.3 \%)$. Business owners were $21(19.8 \%)$ followed retired at $9(8.5 \%)$. Casual laborers were at $9(8.5 \%)$ and lastly petty traders at $6(5.6 \%)\left(\chi^{2}=16.992, \mathrm{p}=0.009, \mathrm{df}=5\right) . \mathrm{P}=0.009$ was lower than $\mathrm{p}=$ 0.05 , chi-square statistics indicates there was a significant relationship between occupation and diarrhea symptoms (Table 1).

Most respondents 33 (30.8\%) earned between $10 \mathrm{k}-30 \mathrm{k}$ and were the most statistically significant, followed by those who earned 31 (29.0\%) $30-60$ k. 21 (19.6\%) earned between $60 \mathrm{k}-150 \mathrm{k}$, while $15(14.0 \%)$ earned between $2 \mathrm{k}-10 \mathrm{k}$, 4 (3.7\%) earned between $150 \mathrm{k}-200 \mathrm{k}$ and lastly $3(2.8 \%)$ earned less than $2 \mathrm{k}$. $\left(\chi^{2}=2.264, \mathrm{p}=0.812, \mathrm{df}=5\right) . \mathrm{P}=0.812$ was higher than $\mathrm{p}=0.05$, chi-square statistics indicates there was no significant relationship between income status and diarrhea symptoms (Table 1).

\subsection{Prevalence of Food Poisoning}

A total of 1000 patients were observed in Thika level 5 hospital with signs of food poisoning. Out of 1000, according to labolatory test results 623 (62.3\%) were total positive for diarrhea while 377 (37.7\%) were total negative.623 (62.3\%) was the number that was used for further analysis of bacterial agents because they were the only number that turned positive according to test strips for diarrhea. Further computation using the 2 by 2 contingency table revealed the following truth, total diseased number was 587 while non-diseased number was 413. Sensitivity of the labolatory tests revealed that the true positives were 228 while false positive was 395 . Specificity of the labolatory tests revealed true negatives were 18 while false negatives were 359 .

$$
\text { Prevalence }=(\text { total }- \text { diseased } / \text { total }) \times 100=(587 / 1000) \times 100=58.7 \%
$$

Positive prevalence was therefore 58.7.

\subsection{Bacterial Causative Agents of Food Poisoning}

\section{Stool Analysis Results}

A total of 1000 patients came to the clinic and out of them 623 (62.3\%) were positive for diarrhea. $460(73.8 \%)$ were salmonella samples while $164(26.3 \%)$ were cholera. Using proportional probability so 28 patients with vibrio cholera and 78 patients with salmonella species were included in the study (Figure 1). Cholera was caused by the serotype Ogawa $(\mathrm{AB})$ and of one biotype El tor of toxigenic group $1 \mathrm{~V}$ Cholera. The serogroup was from O139 initially isolated from China. Vibrio cholera is a gram-negative, highly motile curved rod with a single polar flagellum. Salmonellosis was caused by salmonella streptococcus isolated from stool culture tests. It is a genus of rod shaped bacteria of the 


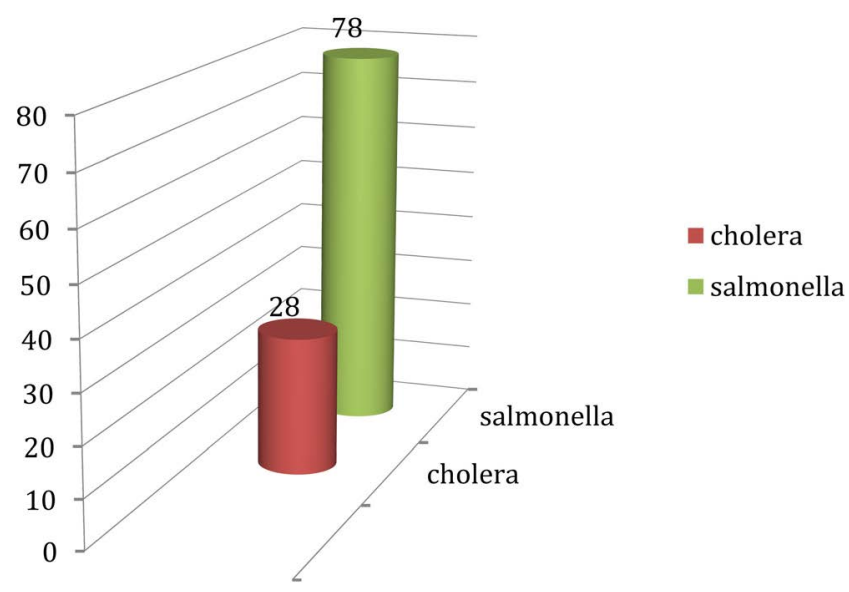

Figure 1. Stool analysis results.

enterobacteriacae family of which two species exist Salmonellabongori and Salmonella enterica. The typhoidal serotypes common in sub-Saharan Africa invaded the gastrointestinal tract of these respondents and they therefore were put on anti-biotics.

\section{Discussion}

\subsection{Socio-Demographic and Socioeconomic Factors}

The findings from this study showed that socio-demographic and economic factors mainly education, gender, age, income level, occupation, residence influenced food poisoning. These findings contradict those of Root [11] who found out that the level of women's education in particular is often strongly associated with child health, higher levels of education lead to improved care seeking behavior, proper hygiene and a better understanding of causes of diarrheal diseases among children.

Findings from this study contradict those of Nsagha [12] who found that there were more females who had cholera than males. Most women engage in more activities which expose them to this infection.

Findings from this study also support those of Valcin [13] who found out that the leading age group is adults (21 - 40 ) years of age, whereas children who were less than or equals to $\leq 20$ years of age had a high percentage of diarrhea perhaps because of rotavirus or other childhood diarrheal illness in addition to bacterial infections.

Findings from this study contradict those of Root [11] who found that income allows households to purchase proper and adequate housing, improve drinking water and an upgrade to sanitation systems. When several households are able to afford such improvements, risk of cross-household contamination is even less. Higher income households are more likely to have a latrine with a septic system that is cement ring latrines with septic holding tank) or a deep tube well, both of which protect household members from faecal-oral contamination.

Findings from this studies also supported those of Nsagha [12] who found out 
that Most women engage in more activities which expose them to this infection in as much as men take part in activities that may involve a lot of manual work, this together with poor hygiene practices contribute to food poisoning.

Findings from this study also contradict those of Sur [14] who found that residence has no substantial relationship to amount of food poisoning. Majority of food poisoning cases came from the peri-urban slums and was influenced by poor infrastructures and poor drainage systems.

\subsection{The Prevalence of Food Poisoning}

A total of 1000 patients were observed in Thika level 5 hospital with signs of food poisoning. Out of 1000, according to labolatory test results 623 (62.3\%) were total positive for diarrhea while 377 (37.7\%) were total negative. 623 $(62.3 \%)$ was the number that was used for further analysis of bacterial agents because they were the only number that turned positive according to test strips for diarrhea. Further computation revealed that 587 were diseased while non-diseased number was 413 . Sensitivity of the labolatory tests revealed that the true positives were 228 while false positive was 395 . Specificity of the labolatory tests revealed true negatives were 18 while false negatives were 359 . The prevalence was $58.7 \%$.

\subsection{Bacterial Causative Agents}

Using proportional probability 28 patients with vibrio cholera and 78 patients with salmonella species were included in the study. Cholera was caused by the serotype Ogawa (AB) and of one biotype El tor of toxigenic group $1 \mathrm{~V}$ Cholera. The serogroup was from O139 initially isolated from China. There are $>200$ serogroups of Vibrio cholerae that are distinguished by the polysaccharides somatic (O) antigen. Of these, only 2 serogroups are toxigenic: $\mathrm{O} 1$ and O139. O139 causes epidemic disease. Serogroup O1 has 2 biotypes, El Tor and classical 01. Both of these biotypes can be further classified in 2 cross-reacting serotypes, Ogawa and Inaba. Compared to the classical 01 strains, El Tor persists longer in the environment, is associated with a higher rate of asymptomatic or mild cases and is shed for longer and in higher numbers in the faeces. In 1992, O139, emerged in South Asia and has been circulating there but has not spread globally. Another variant of El Tor has emerged in the last 10 years and has replaced the original El Tor strain in several parts of Asia and Africa. Vibrio cholera is a gram-negative, highly motile curved rod with a single polar flagellum. It occupies various aquatic environments in various geographic locations. It demonstrates a greater affinity for warmer more tropical climates. It is found in a range of mostly saltwater, and a few freshwater, environs, ubiquitous in marine environment and are frequently found in fish, especially filter-feeding shellfish for example oysters, clams, cockles and can demonstrate very high levels of colonization. This environment is a potentially important reservoir for human infection in developed countries. Salmonellosis was caused by Salmonella streptococcus isolated from stool culture tests. It is a genus of rod shaped bacteria of the 
enterobacteriacae family. Two species exist Salmonellabongori and Salmonella enterica. The typhoidal serotypes common in sub-Saharan Africa invaded the gastrointestinal tract of these respondents and they therefore were put on anti-biotics.

\section{Conclusion}

The true prevalence of food poisoning was $58.7 \%$ in Kiambu County. Food poisoning was largely from the Salmonella bacteria with bloody diarrhoea. Vibrio cholera also contributed to poisoning albeit at a low level.

\section{Limitation of the Study}

Reagents \& equipment sometimes ran out so we had to wait for re-stocking. This of course increased the time of study but we managed to eventually finish the research.

\section{Acknowledgements}

We are grateful to all the patients for their co-operation during sample collection. We also wish to acknowledge the Management of Thika level 5 Hospital for allowing us to do this work in their facility.

\section{Conflicts of Interest}

The authors declare no conflicts of interests regarding the publication of this paper.

\section{References}

[1] Lee, H.-L., Lin, H.-J., Yeh, S.T.-Y., Chi, C.-H., Guo, H.-R., Watson, W., et al. (2008) Presentations of Patients of Poisoning and Predictors of Poisoning-Related Fatality: Findings from a Hospital-Based Prospective Study. BMC Public Health, 8, 7. https://doi.org/10.1186/1471-2458-8-7

[2] The Millennium Development Goals Report 2015.

[3] Mclinden, T., Sargeant, J.M., Thomas, M.K., Papadopoulos, A. and Fazil, A. (2014) Component Costs of Foodborne Illness: A Scoping Review. BMC Public Health, 14, 509. https://doi.org/10.1186/1471-2458-14-509

[4] Gumbo, A., Bangure, D., Gombe, N.T., Mungati, M., Tshimanga, M., Hwalima, Z., et al. (2015) Staphylococcus aureus Food Poisoning among Bulawayo City Council Employees, Zimbabwe, 2014. BMC Research Notes, 8, 485. https://doi.org/10.1186/s13104-015-1490-4

[5] WHO-2015 (2015) Who Executive Summary of Food Borne Diseases 2016. WHO Executive Summary of Food Borne Diseases 2016.

[6] Todd, E.C. (1997) Epidemiology of Foodborne Diseases: A Worldwide Review. World Health Statistics Quarterly. Rapport Trimestriel de Statistiques Sanitaires Mondiales, 50, 30-50.

[7] Baradaran Moghaddam, A., Alebouyeh, M., Farzi, N., Bayati, S. and Amirmozafari, N. (2016) Sensitivity to Nitazoxanide among Metronidazole Resistant Helicobacter pylori Strains in Patients with Gastritis. Medical Journal of the Islamic Republic of 
Iran, 30, 405.

[8] Ombui, J.N., Kagiko, M.M. and Arimi, S.M. (2001) Foodborne Diseases in Kenya. East African Medical Journal, 78, 40-44. https://doi.org/10.4314/eamj.v78i1.9111

[9] Oey, M. and Malhotra, P. (2016) Azithromycin and Ceftriaxone Combination Treatment for Relapsed Salmonella Paratyphi A Bacteraemia. Journal of Travel Medicine, 23, tav032. https://doi.org/10.1093/jtm/tav032

[10] Dupke, S., Akinsinde, K.A., Grunow, R., Iwalokun, B.A., Olukoya, D.K., Oluwadun, A., et al. (2016) Characterization of Vibrio cholerae Strains Isolated from the Nigerian Cholera Outbreak in 2010. Journal of Clinical Microbiology, 54, 2618-2621. https://doi.org/10.1128/JCM.01467-16

[11] Root, E.D., Rodd, J., Yunus, M. and Emch, M. (2013) The Role of Socioeconomic status in Longitudinal Trends of Cholera in Matlab, Bangladesh, 1993-2007. PLoS Neglected Tropical Diseases, 7, e1997. https://doi.org/10.1371/journal.pntd.0001997

[12] Nsagha, D.S., Atashili, J., Fon, P.N., Tanue, E.A., Ayima, C.W. and Kibu, O.D. (2015) Assessing the Risk Factors of Cholera Epidemic in the Buea Health District of Cameroon. BMC Public Health, 15, 1128. https://doi.org/10.1186/s12889-015-2485-8

[13] Valcin, C.-L., Severe, K., Riche, C.T., Anglade, B.S., Moise, C.G., Woodworth, M., et al. (2013) Predictors of Disease Severity in Patients Admitted to a Cholera Treatment Center in Urban Haiti. The American Journal of Tropical Medicine and Hygiene, 89, 625-632. https://doi.org/10.4269/ajtmh.13-0170

[14] Sur, D., Deen, J.L., Manna, B., Niyogi, S.K., Deb, A.K., Kanungo, S., et al. (2005) The Burden of Cholera in the Slums of Kolkata, India: Data from a Prospective, Community Based Study. Archives of Disease in Childhood, 90, 1175-1181.

https://doi.org/10.1136/adc.2004.071316 